\title{
A New Formation Process of Poly(phenylsilsesquioxane) in the Hydrolytic Polycondensation of Trichlorophenylsilane. Isolation of Low Molecular Weight Hydrolysates to Form High Molecular Weight Polymers at Mild Reaction Conditions
}

\author{
Eung-Chan LEE and Yoshiharu KIMURA \\ Department of Polymer Science and Engineering, Kyoto Institute of Technology, \\ Matsugasaki, Sakyo-ku, Kyoto 606, Japan
}

(Received January 14, 1997)

\begin{abstract}
Trichlorophenylsilane (TCP) was hydrolyzed in an toluene/water heterogeneous solvent system, and a low molecular weight hydrolysate with simple structure was isolated from the aqueous solution while a high molecular weight hydrolysate with complicated structure was isolated from the toluene layer. When this hydrolysis was conducted strictly at $0{ }^{\circ} \mathrm{C}$, the primary hydrolysate consisting mainly of phenylsilanetriol (PST) could be crystallized out from the aqueous layer. When the low molecular weight hydrolysate obtained from the aqueous layer was subjected to the $\mathrm{KOH}$-catalyzed polycondensation in refluxing toluene, poly(phenylsilsesquioxane) (PPQS) with a highly ordered ladder structure could be obtained. Its molecular weight increased with decreasing molecular weight of the initial hydrolysate, and its silanol content oppositely decreased with it. Since this polycondensation proceeded stepwise, it has been considered that intramolecular cyclization is favored in the first condensation of the low molecular weight hydrolysate and that the resultant higher oligomers are then connected into high polymer with ladder structure by the silanol coupling. The molecular weight of PPQS finally obtained reached $120 \mathrm{kDa}$.

KEY WORDS Trichlorophenylsilane / Phenylsilanetriol / Hydrolytic Polycondensation / Poly(phenylsilsesquioxane) / Ladder Structure /
\end{abstract}

Poly(phenylsilsesquioxane) (PPQS: 1), consisting of a siloxane ladder (Scheme 1), is known to have various potential applications because of their supreme thermal and electrical properties. ${ }^{1}$ However, the real application has been interfered by its brittle nature and difficult control of its structure and properties. PPQS has usually been prepared by hydrolytic polymerization of trichlorophenylsilane (TCP) and trialkoxyphenylsilanes. Simple hydrolysis of these compounds, however, gives an oligomeric condensate having molecular weight of $1000-4000 \mathrm{Da}^{2}$ instead of monomeric phenylsilanetriol (PST). When this oligomer is subjected to the ordinary base-catalyzed dehydrative condensation using toluene as the solvent, the resultant PPQS should have many defects in its ladder structure with limited increase in molecular weight. It has been known, however, heattreatment of this polymer in solid state at high tem-

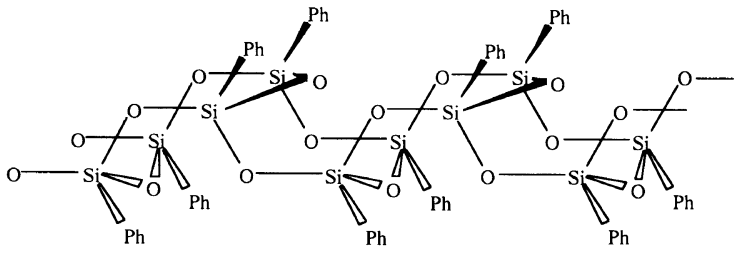

1a (trans - sydiotactic double chain)

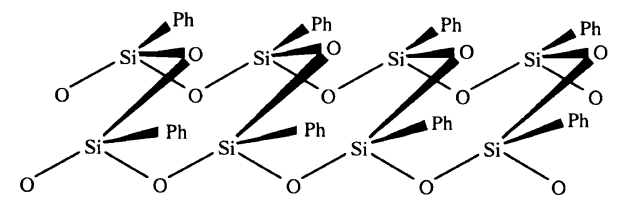

$1 \mathrm{~b}$ (cis - isotactic double chain)

Scheme 1. perature, for example, at $300-400^{\circ} \mathrm{C}$, increases the molecular weight up to $10-100 \mathrm{kDa}$ without losing solubility of the product. ${ }^{3}$ The high polymer thus obtained is thought to keep the ladder structure although neither the chain regularity or the terminal structure has been analyzed.

Brown reported in his pioneering work $^{3,4}$ that the cyclic tetramers 2 with eight-membered ring are formed by dehydration of PST and that these tetramers are connected in the following step to form PPQS with ladder structure (Scheme 2). They also pointed out that the ladder of PPQS is in equilibrium with cage compounds 3 under the basic conditions and that the molecular weight can be increased only by solid state polymerization. However, this mechanism should be inconsistent
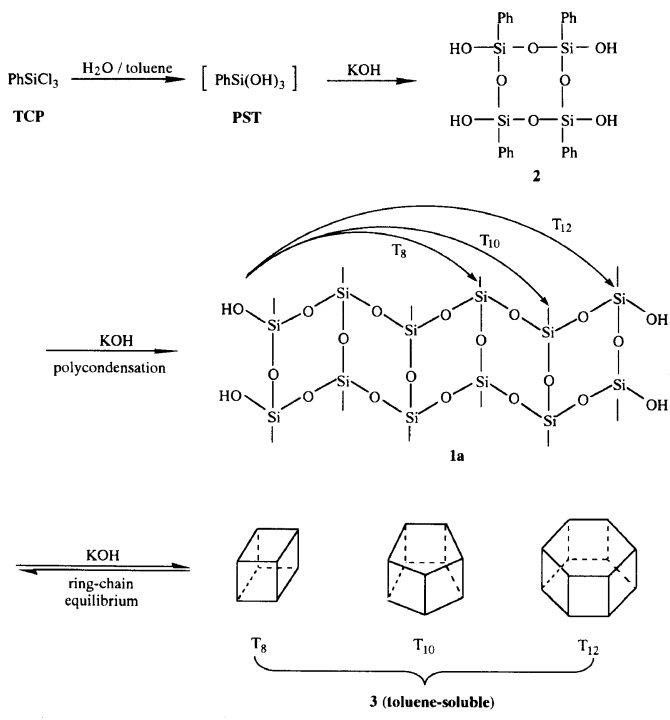

Scheme 2. 
with the initial formation of prepolymer which has generally been observed in the step of hydrolysis of TCP as described earlier. The formation of the key intermediates PST and the cyclic tetramer has not been confirmed, either. In 1959,5 Takiguchi reported the isolation of PST by the hydrolysis of TCP under completely different reaction conditions where aniline was utilized as trapping agent of hydrochloric acid. The crystalline PST thus isolated, however, was insoluble in water and gave only an oligomeric product when reacted in the basic conditions. Recently, ${ }^{6}$ Lichtenhan succeeded in isolating a new polycyclic system of oligo(siloxane) from acidic aqueous solution of the hydrolysate of TCP, which suggests a different reaction course present in the formation of the PPQS precursor. More recently, ${ }^{7}$ Hayashida et al. analyzed the condensation pathway from the oligomeric hydrolysate of TCP to PPQS by ${ }^{29} \mathrm{Si}$ NMR spectroscopy. It was suggested that the terminal silanol groups are likely involved in the formation of cyclics. In spite of these facts the formation mechanism of PPQS is still controversial, and how the TCP hydrolysate is formed or how the ladder can be formed from it is not well understood. Consequently, we decided to analyze the hydrolysis of TCP for characterzation of its initial hydrolysate.

In this paper we report on the structure and polymerizability of various types of TCP hydrolysates which are obtained when TCP is hydrolyzed in a water/ toluene solvent system at different reaction conditions. It has been found that low molecular weight hydrolysates can be isolated from the aqueous layer and that their base-catalyzed polycondensation can afford high molecular weight PPQS.

\section{EXPERIMENTAL}

\section{Materials}

TCP was supplied by Nacalai Tesque (Kyoto) and purified by distillation. Toluene was distilled over sodium metal before use. Other solvents and reagents were commercially supplied and used without purification.

\footnotetext{
Measurements

${ }^{29} \mathrm{Si}$ NMR spectra were measured at $59.7 \mathrm{MHz}$ on a GE QE-300 spectrometer in acetone- $d_{6}$ and toluene- $d_{8}$ with a $1 \mathrm{vol} \%$ of tetramethylsilane (TMS) in $\mathrm{CDCl}_{3}$ as the external standard. The sample was put in an NMR tube made of glass $(5 \mathrm{~mm} \phi)$ which was rotated at $5 \mathrm{kHz}$ at a constant temperature of $21^{\circ} \mathrm{C}$ throughout the experiment. ${ }^{1} \mathrm{H}$ NMR spectra were measured at $200 \mathrm{MHz}$ on a Varian XL-200 spectrometer in $\mathrm{CDCl}_{3}$ with the solvent peak as the internal reference $(\delta 7.26)$. The number average molecular weight $\left(M_{n}\right)$ and the molecular weight distribution $\left(M_{w} / M_{n}\right)$ were determined by gel permeation chromatography (GPC). The analyzer was composed of a Shimadzu LC-10A pump, a Shodex RI SE-31 RI detector, a Shimadzu C-R7A Chromatopac data processor, a Shodex DEGAS KT-16 degassor, and a Sugai U-620 column oven. A combination of two polystyrene gel columns of Tosoh TSKgel $\mathrm{G}_{4000 \mathrm{H}_{8}}$ and $\mathrm{G} 2500 \mathrm{H}_{8}$ was used with chloroform as the eluent at $35^{\circ} \mathrm{C}$. Its limited exclusion molecular weight was $7 \times 10^{5} \mathrm{Da}$. The molecular weight was calibrated accord-
}

ing to polystyrene standards. FT-IR spectra were recorded on a JASCO FT-IR-5300 spectrophotometer by the $\mathrm{KBr}$ pellet method.

\section{Hydrolysis}

A typical example was as follows. A solution of $6.3 \mathrm{~g}$ of TCP in $163 \mathrm{ml}$ of toluene was added dropwise to $815 \mathrm{ml}$ of water under vigorous agitation at $0^{\circ} \mathrm{C}$ for a period of $1 \mathrm{~h}$. After the addition, the mixture was kept stirred at the same temperature for $30 \mathrm{~min}$, and the aqueous and toluene layers were separated at $0^{\circ} \mathrm{C}$. Then, the $\mathrm{pH}$ of the aqueous layer was adjusted to 4 by addition of an aqueous $\mathrm{NaHCO}_{3}$ solution and kept in a refrigerator at $0^{\circ} \mathrm{C}$ overnight. The hydrolysate, having precipitated out, was isolated by filtration, washed with an ice-water, and dried in vacuo. The toluene layer separated was evaporated to dryness at reduced pressure to obtain the product as had been done in the former studies. ${ }^{2-5}$

For obtaining the hydrolysates with lower molecular weight, all the operations were done at $0^{\circ} \mathrm{C}$. Particularly, the TCP solution in toluene was cooled to $0^{\circ} \mathrm{C}$ during its addition from the dropping funnel, and the filtration and drying of the product precipitated were done in a cold room maintained at $0^{\circ} \mathrm{C} .^{3,4,7}$

\section{Polycondensation}

$5.0 \mathrm{~g}$ of the TCP hydrolysate and $5.0 \mathrm{mg}$ of $\mathrm{KOH}$ $(0.1 \mathrm{wt} \%$ relative to the hydrolysate) were mixed with $38 \mathrm{ml}$ of toluene. The mixture was heated at the refluxing temperature of toluene for $16 \mathrm{~h}$ with the dehydrated water removed from the azeotropically distilled toluene/ water mixture in a Dean-Stark tube. The viscous solution finally obtained was poured into a large excess of methanol to precipitate out the condensation product. The product was then filtered and dried in vacuo at $150^{\circ} \mathrm{C}$ for $10 \mathrm{~h}$.

\section{RESULTS AND DISCUSSION}

In the ordinary synthesis of PPQS, TCP is first hydrolyzed in an organic/aqueous two-phase solvent system, and its hydrolysis product is separated from the organic layer and subjected to polycondensation by the catalysis of $\mathrm{KOH}$ in refluxing toluene. Brown suggested ${ }^{3}$ that the TCP hydrolysate obtained from the organic (toluene) layer should be a primary condensate of PST, comprising such cyclics as $\mathrm{T}_{4}(2)$ and $\mathrm{T}_{8}(3)$. The other researchers such as Andrianov ${ }^{8}$ and Chojnowski ${ }^{9}$ also stood on the Brown's reaction scheme, although they believed that the hydrolysate of TCP consists of a partial ladder structure. Other researchers showed that the TCP hydrolysate contains a significant amount of silanol groups with wide molecular weight distribution around an average molecular weight of $2-4 \mathrm{kDa} .^{10,11}$ In these studies, however, the yield of the hydrolysate was always lower than the theoretical value, sometimes less than $80 \%{ }^{11}$ This may suggest that some of the hydrolysate remained in the aqueous layer without being extracted with the organic solvent. Since the primary hydrolysate of TCP should be PST or its primary condensation product, it should involve many hydrophilic silanol groups and therefore more soluble in aqueous layer than in organic layer if no further condensation 
would take place. So, we first analyzed how much hydrolysis product can be obtained from the aqueous layer and how low the molecular weight can be retained in it by changing the hydrolysis conditions.

\section{Isolation of Hydrolysate from Aqueous Layer}

TCP was hydrolyzed by dropwise addition of its toluene solution into a water at $0^{\circ} \mathrm{C}$ with a water/ toluene volume ratio of $1 / 1$, and after this hydrolysis the aqueous layer was separated from the toluene layer. While the toluene-soluble hydrolysate was readily isolated by evaporating the solvent, the water-soluble hydrolysate was not easily isolated from the aqueous layer, because evaporation of water caused dehydration of the product itself. Fortunately, we found that the hydrolysate could precipitate out in solid form when the aqueous layer was kept around $0^{\circ} \mathrm{C}$ after its $\mathrm{pH}$ adjusted to 4.0. It was then readily isolated by filtration. Figure 1 shows the changes in yield and molecular weight of the hydrolysates thus isolated from both layers at different TCP concentrations in toluene. It is known that the yield of the hydrolysate from the aqueous layer increased with decreasing concentration of TCP (shown in $\mathrm{wt}^{\%} \%$ relative to toluene), while the yield from the toluene layer decreased. When the concentration was $0.5 \mathrm{wt} \%$, the yield from the aqueous layer became higher than $40 \%$ out of $70 \%$ in total yield. This may be because a considerable amount of the hydrolysate had still remained in the aqueous solution without precipitating. The other reason for the lower total yield is that the hydrolysate easily reacted with the surface of glass wares to hinder its recovery. It should also be noted that the molecular weight of the hydrolysate isolated from the aqueous layer was always lower than that of the hydrolysate isolated from the toluene layer, but not lower than $500 \mathrm{Da}$

The same hydrolysis was examined at different water/toluene volume ratios with the TCP concentration kept constant at $2.5 \mathrm{wt} \%$ relative to toluene. Figure 2

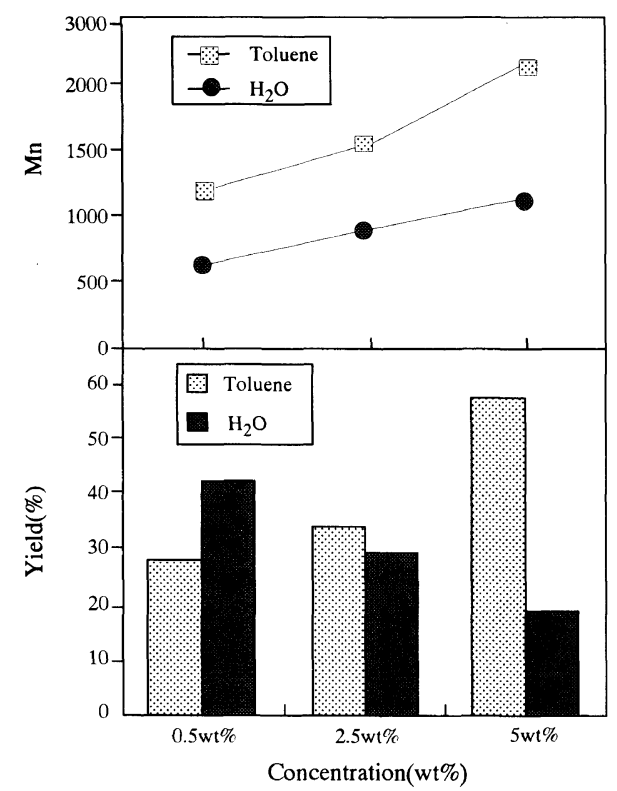

Figure 1. Effect of the TCP concentration on the yields of the hydrolysate from the aqueous and toluene layers at a constant solvent ratio. shows the changes in yield and molecular weight of the hydrolysates isolated from both layers. It is known that the yield from the aqueous layer increased with increasing water/toluene volume ratio, while the yield from the toluene layer decreased with it significantly. As found in the above data, the molecular weight of the hydrolysates isolated from the aqueous layer was much lower than that of the hydrolysates isolated from the toluene layer, being higher than $500 \mathrm{Da}$. These facts suggest that the primary hydrolysate of TCP having enough low molecular weight should be soluble in water and that it should incur dehydrative self-condensation during storage at $0^{\circ} \mathrm{C}$ and $\mathrm{pH}=4$ to have a threshold molecular weight above which the oligomeric products should start precipitation.

In the previous studies, ${ }^{2-10}$ TCP was hydrolyzed in a relatively small volume of water, and its primary hydrolysate would have been easily exposed to the aforementioned oligocondensations to give the toluenesoluble oligomeric products with a relatively high molecular weight. These oligomers may alternatively have been formed by the dehydrochloric condensation between TCP and its primary hydrolysate at the interface of water/toluene solvent system. When the toluene solution of TCP was added to an aqueous solution with its $\mathrm{pH}$ adjusted at $4.0-7.0$ by addition of $\mathrm{NaHCO}_{3}$ solution, the yield of low molecular weight fraction from aqueous layer became less than 15\%. Probably, condensation of PST should have been driven in a faster rate at slightly acidic conditions than at highly acidic conditions. Lichtenhan isolated a polycyclic oligomer after keeping the highly acidic aqueous solution formed by hydrolysis of TCP at room temperature $t$ for a long period, even 1 month. ${ }^{6}$ Therefore, exposure of PST to neutral and basic conditions should bring it fast polycondensation, probably through general base catalysis which is attributed to the conjugate base of salt.

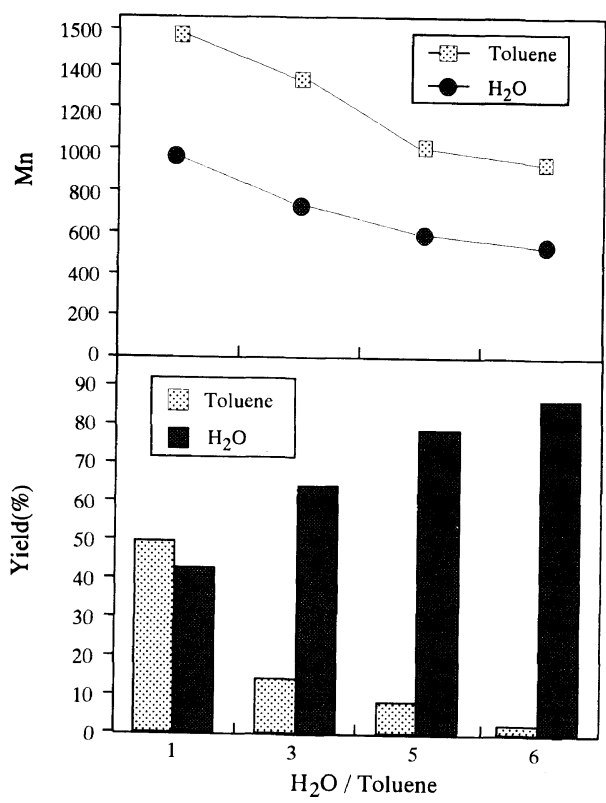

Figure 2. Effect of the $\mathrm{H}_{2} \mathrm{O}$ /toluene volume ratio on the yields of the hydrolysate from the aqueous and toluene layers at a constant TCP concentration. 


\section{Isolation of the Primary Hydrolysate of TCP}

The above data strongly supported the formation of the primary hydrolysate of TCP in the aqueous layer. So, we carefully carried out the TCP hydrolysis and product isolation so as for the reaction system to keep $0^{\circ} \mathrm{C}$ for minimizing the oligocondensation of the primary hydrolysate. In the optimum case the hydrolysate with an average molecular weight of $290 \mathrm{Da}$ by GPC was obtained from the aqueous solution in $70 \%$ yield. However, the molecular weight could not be lowered than this value. In the course of these trials we noticed that a small amount of white crystalline precipitates were formed and settled down in the aqueous layer upon the $\mathrm{pH}$ adjustment to 4 . These precipitates were quickly filtered and dried in a vacuum of $18 \mathrm{mmHg}$ at $0^{\circ} \mathrm{C}$. Although their yield was only $10 \%$ at highest, their average molecular weight was $170 \mathrm{Da}$ as determined by GPC, which happened to be near the real molecular weight of monomeric PST. They decomposed gradually when placed in atmosphere and very quickly when heated.

Figure 3 shows the ${ }^{1} \mathrm{H}$ NMR spectra of the hydrolysates with different average molecular weight. The proton signal of silanol can be detected around $2-3 \mathrm{ppm}$, and its signal width is sharper in the product with lower molecular weight. The complex signals due to the phenyl protons are also significantly different among the three. The integral ratio of the phenyl and silanol signals was almost 5.0/2.0 irrespective to the molecular weight of the products, although the signal intensity of the silanol proton seems to be somewhat arbitrary due to the different relaxation time or signal broadening of the protons in strong hydrogen bonding and in different environment. Considering that the same ratio should be $5 / 3$ in PST, a part of the silanol groups of PST is thought to be dehydrated for condensation even in the hydrolysate having the lowest average

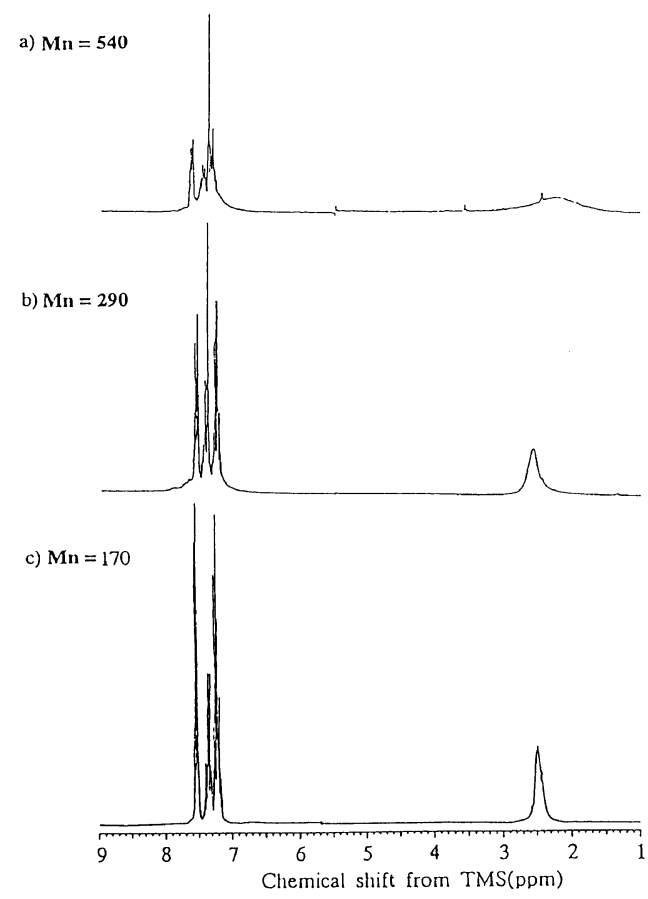

Figure 3. ${ }^{1} \mathrm{H}$ NMR spectra of the hydrolysates with a) $M_{n}=540$, b) $M_{n}=290$, and c) $M_{n}=170$ in $\mathrm{CDCl}_{3}$. molecular weight. In the IR spectra, the hydrolysate with $M_{n}=170$ showed a small absorption due to $\mathrm{Si}-\mathrm{O}$ stretching at $1130 \mathrm{~cm}^{-1}$, while the products with higher molecular weights showed the strong, broad band due $e^{7,10}$ to $\mathrm{Si}-\mathrm{O}$ stretching at $1100 \mathrm{~cm}^{-1}$. These data strongly support that the former hydrolysate comprises PST as the main product.

Figure 4 shows their ${ }^{29} \mathrm{Si}$ NMR spectra in acetone- $d_{6}$. The hydrolysate with $M_{n}=170$ shows a signal at $-71.6 \mathrm{ppm}$ with a shoulder peak at a slightly higher field $(72.4 \mathrm{ppm})$. The hydrolysates with higher molecular weight, in return, show complex signals at higher fields probably due to their different degree of oligomerization. ${ }^{7,12}$ These data also support that the hydrolysate with $M_{n}=170$ consists mainly of PST in spite of slight contamination with dimer and/or trace of trimer and that the hydrolysates with higher molecular weight are oligocondensates of PST. The fact that the former hydrolysate crystallized out from the aqueous layer on $\mathrm{pH}$ adjustment suggests that PST is the primary hydrolysate of TCP and that PST should be soluble in the highly acidic aqueous solution resulting with the TCP hydrolysis but less soluble in the salt-containing solution resulting with the $\mathrm{pH}$ adjustment. Its small yield may be attributed to its aforementioned oligocondensation during the hydrolysis of TCP. Therefore, it should be very difficult to stop the intermolecular condensation of PST in the aqueous solution. Once the hydrolysis products are isolated from the aqueous solution, the rate of their silanol condensation should be much slower.

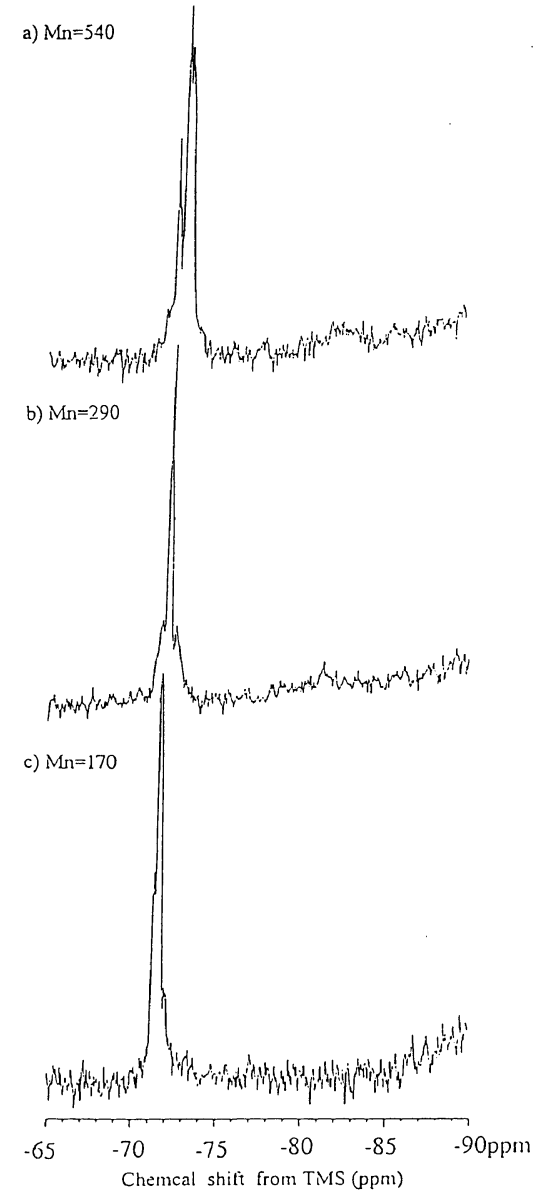

Figure 4. ${ }^{29} \mathrm{Si}$ NMR spectra of the hydrolysates with a) $M_{n}=540$, b) $M_{n}=290$, and c) $M_{n}=170$ in acetone- $d_{6}$. 
Effect of the Molecular Weight of the Hydrolysate on the Polycondensation

With the hydrolysates obtained above, polycondensation was examined in refluxing toluene with $0.1 \mathrm{wt} \%$ of $\mathrm{KOH}$ as catalyst. ${ }^{3,4}$ The resulting polycondensates were dried at $150^{\circ} \mathrm{C}$. We confirmed that this drying condition gave no structure change on the products. The alternative acid-catalyzed polycondensation of the hydrolysates gave only insoluble products in most cases.

Figure 5 shows the changes in average molecular weight of the resultant polycondensates with the reaction time for the three hydrolysates. It is known that the molecular weight of the polycondensates increased with increasing reaction time and that the increase was larger with decreasing molecular weight of the starting hydrolysates. Particularly, the hydrolysate with $M_{n}=170$ gave the highest molecular weight of $120 \mathrm{kDa}$ after reaction for $16 \mathrm{~h}$. The ${ }^{29} \mathrm{Si}$ NMR spectrum of this polycondensate in toluene- $d_{8}$ showed a broad peak at $-87 \mathrm{ppm}^{7,12}$ with a slightly high level of noise. Since the chemical shift of this signal is much higher in magnetic field than those of the signals of the hydrolysates (Figure 4 ), the silicon atoms of the polycondensate are considered to be trigonally bound with oxygen atoms ( $\mathrm{T}_{3}$ form) to form the ring structure similar to that $\left(\mathrm{Q}_{4}\right.$ form) of the silicon oxide unit in glass. ${ }^{13}$ This result indicates that the polycondensate has the ladder structure of $\mathbf{1}$, although its conformation is uncertain. Since the polycondensates with lower molecular weights showed similar spectra, the ${ }^{29} \mathrm{Si} \mathrm{NMR}$ spectrum is not so sensitive to the micro structure of the ladder.

Figure 6 shows the IR spectra of the polycondensates finally obtained. The two strong absorptions characteristic of the ladder siloxane bond ${ }^{3,4}$ can be detected around $1100 \mathrm{~cm}^{-1}$ in every case. In the spectra of the high molecular weight polycondensates prepared from the hydrolysates with $M_{n}=290$ and 170, the absorption due to silanol (at $3360 \mathrm{~cm}^{-1}$ ) is very weak, while it is strong in the spectrum of the low molecular weight polycondensate which was prepared from the hydrolysate with $M_{n}=540$. When the hydrolysates obtained from the toluene layer were subjected to the identical polycondensation, the products had a molecular weight from 10 to $20 \mathrm{kDa}$, showing an IR spectrum similar to the latter. Therefore, the polycondensates obtained from the hydrolysates with higher molecular weights have many

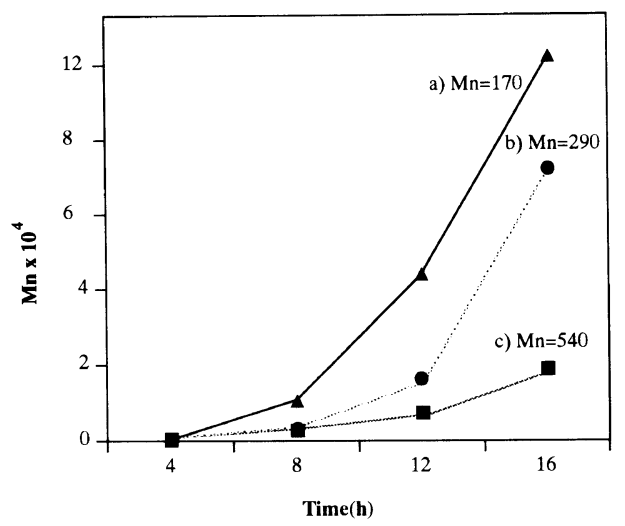

Figure 5. Increase in molecular weight of PPQS with reaction time in the polycondensation of the hydrolysates with the $M_{n}$ values indicated. silanol groups that are isolated from each other by the local crosslinked networks. This means that many defects and chain branches should exist in their ladder structure.

Figure 7 shows the time-dependent changes in GPC curve of the products in the polycondensation of the hydrolysates with $M_{n}=170$ and 540 . It is clearly shown that the peak shifted to the higher molecular weight range with the reaction proceeding. In the case of the hydrolysate with $M_{n}=170$ the initial polycondensate formed after $4 \mathrm{~h}$ had an average molecular weight of 460 with a wide molecular weight distribution. After $8 \mathrm{~h}$ the product showed two peak tops at 800 , and $1400 \mathrm{Da}$. Then the peak shifted to higher molecular weight regions with the bimodal feature kept. The molecular weight distribution became relatively sharp compared to that observed in the ordinary polycondensates. On the other hand, the hydrolysate with $M_{n}=540$ showed a bimodal curve which is quite different from the curve of the corresponding oligomer obtained after the base-catalyzed polycondensation of the hydrolysate with $M_{n}=170$ for $4 \mathrm{~h}$. The curve gradually shifted to the higher molecular weight region with small change in curvature until the average molecular weight increased up to about 12000 with the gradual condensation.

It has been known $^{3}$ that the hydrolysates isolated from the toluene layer can give polycondensates with a molecular weight higher than $10-20 \mathrm{kDa}$ by the similar base-catalyzed polycondensation. Their molecular weight can be increased to several hundred $\mathrm{kDa}$ by the following solid state post-polymerization ${ }^{4}$ where the chain extension should occur by the reaction among the silanol groups placed on the defect points of the ladder and the siloxane networks can also be rearranged to increase the

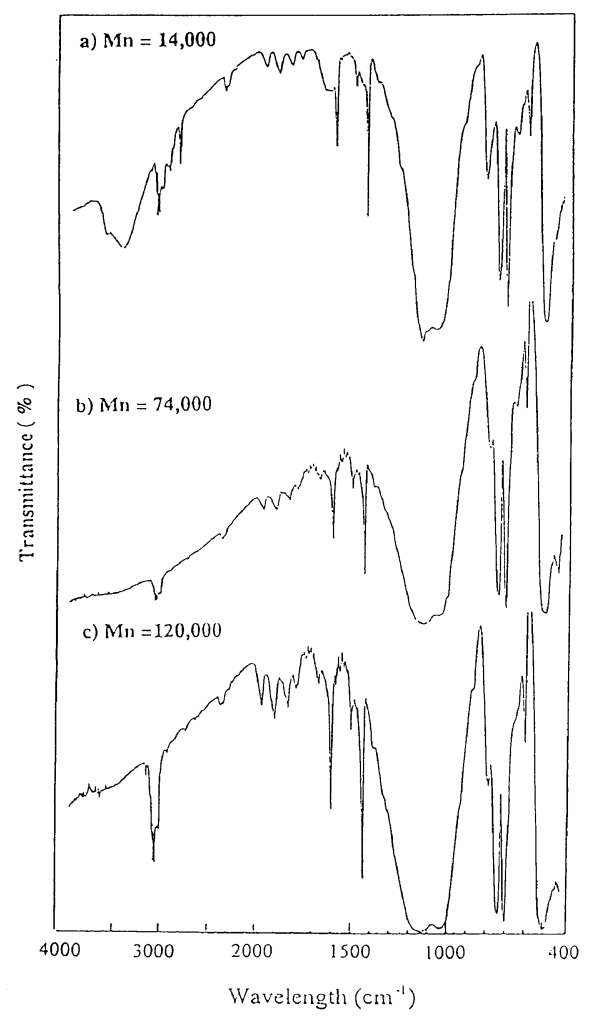

Figure 6. FT-IR spectra of the polycondensates finally obtained from the hydrolysates with a) $M_{n}=540$, b) $M_{n}=290$, and c) $M_{n}=170$. 
a) $\mathrm{Mn}=\mathbf{1 7 0}$

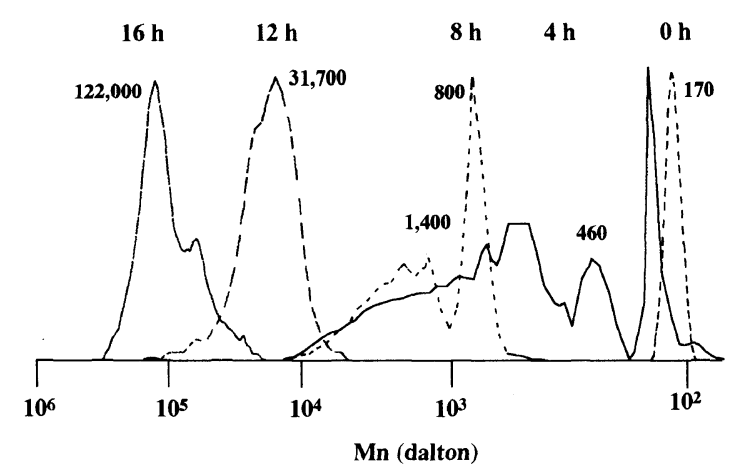

b) $\mathrm{Mn}=\mathbf{5 4 0}$

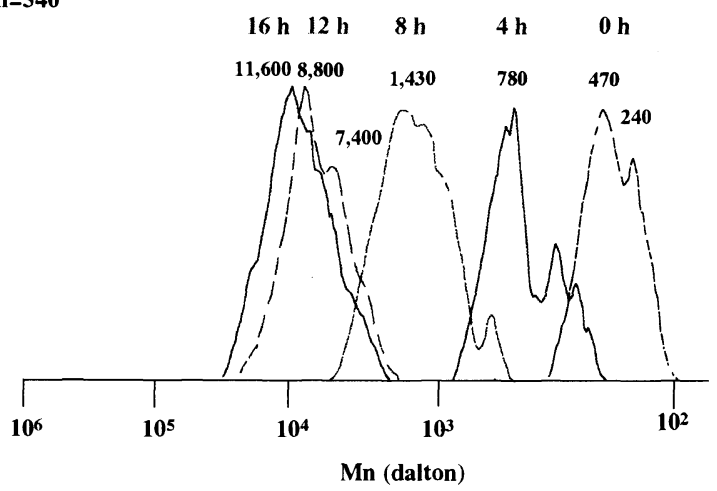

Figure 7. Time-dependent changes in GPC curve of product in the base-catalyzed polycondensation of the hydrolysates with $M_{n}=170$ and 540 .

thermally more stable ladder units. In the present study, the hydrolysates with molecular weight higher than 500 gave an almost identical result, even though they had been isolated from the aqueous solution. The hydrolysates with lower molecular weights, in return, gave polycondensates having enough high molecular weight polycondensates without the solid state post-polymerization. For these polycondensates further increase in molecular weight was not observed even after their solid state post-polymerization at $400^{\circ} \mathrm{C}$ probably because they should have a very small number of silanol groups in the chain.

\section{Reaction Course in the Hydrolytic Condensation of TCP}

Based on the above results, the reaction course of the hydrolytic polymerization of TCP can be deduced as follows (Scheme 3). The hydrolysis of TCP may take place in the toluene/water interface to produce the primary hydrolysate consisting mainly of PST. It is soluble in the aqueous layer, but precipitates out in some degree as crystals upon $\mathrm{pH}$ adjustment. In this primary hydrolysate the dimer and/or trimer (4: $m=2$ or 3$)$ are usually involved because the unstable PST should incur spontaneous dehydrative condensation by itself. When the aqueous solution containing this primary hydrolysate is kept at $0^{\circ} \mathrm{C}$ and at $\mathrm{pH}=4$, its condensation takes place to form the oligomeric hydrolysate (loosely crosslinked) with a molecular weight of several hundreds. When the hydrolysis is conducted in a concentrated solution and when the reaction system is slightly warmed up above $0^{\circ} \mathrm{C}$, the hydrolysates with molecular weight higher than $1 \mathrm{kDa}$ can be formed and extracted by toluene.

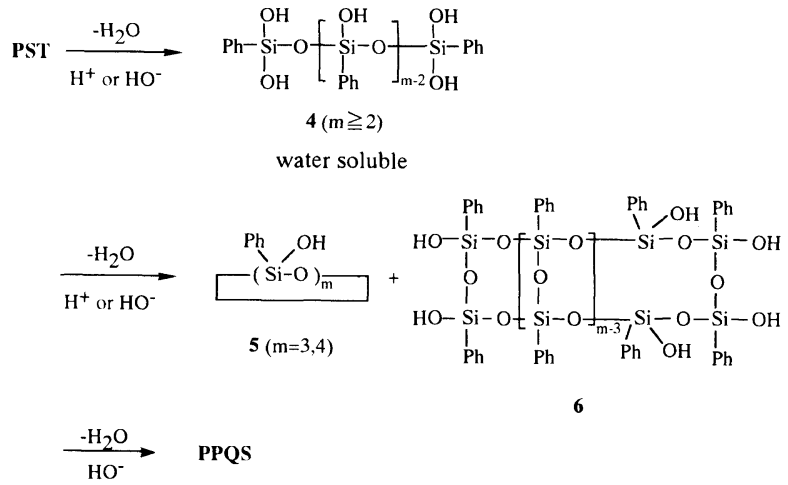

Scheme 3.

The base-catalyzed polycondensation of the hydrolysates also proceeds stepwise with the dehydration of silanol groups, although the each step could not be traced here. First, the primary hydrolysate consisting mainly of PST can be converted to the cyclic oligomers (5) and the oligomers (6) with a loose network system, and then to the ladder polymer. The ladder-ladder coupling is also possible to increase the molecular weight up to $120 \mathrm{kDa}$ in the later stage of polycondensation.

Contrarily, the oligomeric hydrolysates formed in the aqueous solution can be condensed likewise under the basic conditions, leaving many defects in the ladder structure. In this case the starting hydrolysates have a disordered branched structure, and its coupling product should also have a disordered ladder because the rearrangement of the siloxane network once formed is too slow at the present mild reaction conditions. As mentioned above, the solid state polymerization will be needed for increasing its molecular weight and structure regularity. The final structure of the polycondensates is therefore affected by the structure or molecular weight of the starting hydrolysates.

The structure difference between the oligomers formed in the stage of hydrolysis and in the following basecatalyzed condensation system should be attributed to the different molecular arrangements which are allowed at their formation stage. If the condensation is conducted in aqueous environment, the initially formed oligomers should take a conformation like 7 in which the hydrophilic silanols are placed in the outer layer and the hydrophobic phenyl and siloxane chains in the inner core. This conformation is favorable for the intermolecular silanol coupling to drive the disordered chain branching or crosslinking. On the contrary, if the condensation is conducted in organic solvent with the aid of $\mathrm{KOH}$, the phenyl/silanol placement would be turned around. In this arrangement like $\mathbf{8}$, having the silanol groups and catalyst inside the molecular core, the intramolecular cyclization should surpass the intermolecular chain coupling to allow formation of ladder structure until the remaining silanol cannot react any more in the core. After that, the chain extension is allowed through inter-oligomer coupling. The importance of the hydrophilic/hydrophobic balance of the oligomeric hydrolysate on the structure of the poly(silsesquioxane)s is understood considering that the similar hydrolytic polycondensation of methyltrichlorosilane and other analogous compounds which have short alkyl chain can 


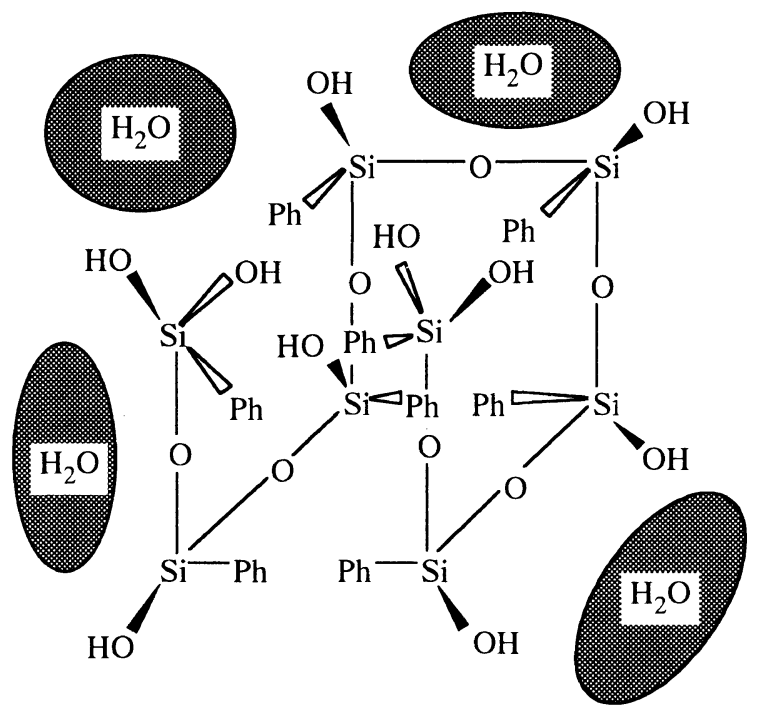

7 (in aqueous solution)

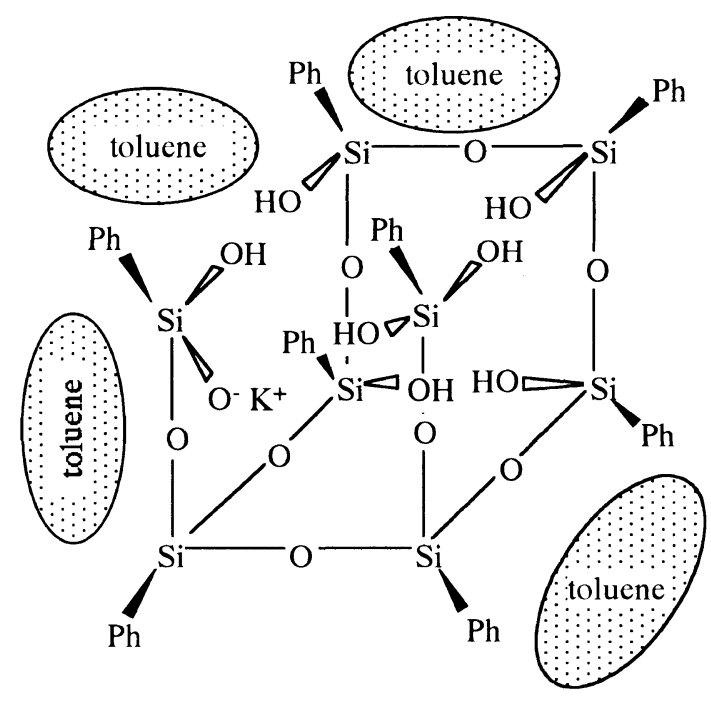

8 (in toluene solution)

Scheme 4.

always form crosslinked polymers without formation of ladder structure. ${ }^{14}$ The formation of the eight membered ring in the ladder structure is probably because it is the most thermally stable with decrease in steric interaction among the bulky phenyl groups. The higher reactivity of the singly bound siloxane units $\left(T_{1}\right)$ relative to the diagonally bound siloxane units $\left(\mathrm{T}_{2}\right)$ can also favor the cylization over the linear chain extension.

At present, we do not have any data on the terminal structure of the polymers and oligomers, and cannot discuss the mechanism of chain extension. However, the formation of the ladder structure suggests that even if the terminal groups are irregular, particularly by the reaction of the cyclic oligomers as $\mathbf{5}(m=3)$, they should be eliminated by the hydration/dehydration equilibrium by the potassium hydroxide catalysis ${ }^{9}$ to lead to formation of well-shaped ladder structure.

\section{CONCLUSION}

In the hydrolysis of TCP a low molecular weight hydrolysate with simple structure could be isolated from the aqueous solution, while a high molecular weight hydrolysate with complicated structure was isolated from the toluene layer. By optimization of the hydrolysis conditions, the primary hydrolysate consisting mainly of PST was allowed to crystallize out from the aqueous layer. When the low molecular weight hydrolysate was subjected to the condensation, PPQS with a well-shaped ladder structure could be obtained. It was found that the molecular weight of PPQS increased with decreasing molecular weight of the hydrolysate. In the present study, the reaction course of the hydrolytic polycondensation from TCP to PPQS has been clearer, but the reac- tion mechanism for the formation of ladder structure is still uncertain.

Acknowledgment. This work was supported by a Grant-in-Aid for Scientific Research in Priority Areas of "New Polymers and Their Nano-Organized Systems" (No. 277/08 10132276) from the Ministry of Education, Science, Sports, and Culture of Japan, which is greatly acknowledged.

\section{REFERENCES}

1. A. Oikawa, S. Fukuyama, and Y. Yoneda, J. Electrochem. Soc., 137, 3223 (1990).

2. T. Takiguchi, R. Fujikawa, Y. Yamamoto, and M. Ueda, Nippon Kagaku Kaishi, 108 (1974).

3. J. F. Brown, J. Polym. Sci., Part C, 1, 83 (1963).

4. J. F. Brown, H. V. Lester, and P. I. Prescott, J. Am. Chem. Soc., 86, 1120 (1963)

5. T. Takiguchi, J. Am. Chem. Soc., 81, 2359 (1959).

6. J. D. Lichtenhan, J. A. Carter, J. W. Gilman, and F. J. Feher, Macromolecules, 26, 2141 (1993).

7. S. Hayashida and S. Imamura, J. Polym. Sci., A, Polym. Chem., 33, 55 (1995).

8. K. A. Andrianov, G. L. Slonimsky, A. A. Zhdanov, D. Ya. Tsvankin, V. Yu. Levin, V. S. Papkov, Yu. P. Kvachev, and E. M. Belavtseva, J. Polym. Sci., 14, 1205 (1976).

9. J. Chojnowski, Spec. Publ. R. Soc. Chem., 166, 59 (1995).

10. J. D. Miller and H. Ishida, Anal. Chem., 57, 283 (1985).

11. H. Nishida, H. Yamane, Y. Kimura, and T. Kitao, Kobunshi Ronbunshu, 53, 193 (1996).

12. H. Ban, A. Tanaka, Y. Kawai, S. Imamura, Polymer, 31, 564 (1990).

13. J. Chojnowski, M. Cypryk, K. Kazmierski, and K. Rozga, J. Non-Cryst. Solids, 125, 40 (1990).

14. J. S. Hrkach and K. Matyjaszewski, J. Polym. Sci., A, Polym. Chem., 33, 285 (1995). 